\title{
Ingenious Method for Conducive Handoff Appliance in Cognitive Radio Networks
}

\author{
J. Josephine Dhivya, M. Ramaswami \\ Department of Computer Applications, Madurai Kamaraj University, India
}

\begin{tabular}{l} 
Article Info \\
\hline Article history: \\
Received Feb 6, 2018 \\
Revised Jul 23, 2018 \\
Accepted Aug 6, 2018 \\
\hline
\end{tabular}

Keyword:

Fitness value

Handoff

Spectrum mobility

\begin{abstract}
Wireless communications deployed in the current epoch claims ceaseless connection among its users thereby leading to the investigation of Cognitive Radio Networks (CRN) which enables to make use of unallocated spectrum optimally and provides uninterrupted connection. Establishing interminable connectivity during the handoff process in spectrum mobility of CRN is a challenging task. This paper elucidates the optimization of handoff process carried out in CRN by incorporating an intelligent method. This includes fuzzy logic wherein the handoff parameters are processed thereby indicating the need of handoff. The proffered method also comprises of a part of genetic algorithm which yields fitness value for reducing the handoff occurrences and enhancing the overall performance of the system is promoted using cuckoo search which decides the mobile node from which the handoff process has to initiate based on the priority generated. This technique ensures that decision is taken ahead of link failure rather than range failure which are the key point in comparison to the existing system. Results obtained through the simulation are satisfactory in terms of delay, throughput, number of failed handoff and handoffs performed in comparison to the existing fuzzy based handoff process in CRN.
\end{abstract}

Copyright (c) 2018 Institute of Advanced Engineering and Science. All rights reserved.

Corresponding Author:

J. Josephine Dhivya,

Department of Computer Applications,

Madurai Kamaraj University,

Madurai - 625021, India.

Email: josedhivya@gmail.com

\section{INTRODUCTION}

The main aim of CRN is facilitating pliant wireless communication by utilizing the available or unallocated spectrum [1]. This is carried out by allowing the unlicensed or secondary user to access the spectrum temporarily wherin the primary user is not deployed [2]. On the arrival of the primary user it is mandatory for the unlicensed user to vacate the access on spectrum thereby switching over to another unoccupied channel to prevent them from harmful interference [3].This process of change over of state or quitting the channel is referred to as handoff [4]. It is essential to note that the handoff process should take place in a swift manner without any hindrance to ensure optimal spectrum handling that is achieved by analyzing handoff performance indicators [5]. One good solution for this would be the choice of deciding the appropriate channel to switch over and the instant at which this action occurs; also choosing a pertinent handoff scheme is mandatory [6].

Among all the available types of handoff mechanism, hybrid intelligent handoff method shows to be quite compromising among the reactive and non-reactive handoff strategies [7]. The proposed methodology is one of the hybrid methods which incorporates fuzzy based genetic algorithm to decide the channel which is already available by predefined rules. Furthermore the handoff process is carried out in a smooth way by extending the fitness value obtained through fuzzy genetic algorithm and using it in the cuckoo search [8] optimization algorithm. The rest of this paper describes the methodology of fuzzy based genetic algorithm 
coupled with cuckoo search for achieving quality handoff procedure along with the simulation and the analysis of handoff performance indicators.

\section{RELATED WORK}

The hybrid methodology of fuzzy with genetic algorithm, though fairly explored considerable works can be found in the literature. In Wanmai and Mingchuan a vertical handoff decision algorithm was proposed to achieve optimum handoff performance in heterogeneous networks [9]. The authors in [10] proposed a new algorithm for handoff optimization in cognitive radio networks by segregating the WRAN into multiple cells. An algorithm for making decision based on multiple criteria was proposed in [11] for channel selection and spectrum decision function. The concept of optimization in cognitive radio with cuckoo search was introduced in [12] which focus on efficient spectrum sensing technique.

The distinctness of our work includes the progression of the handoff process by predicting link failure ahead and incorporating cuckoo search for detecting mobile nodes to initiate the handoff process together with fuzzy genetic system which is an initiative methodology proposed in the literature.

\section{PROBLEM STATEMENT}

The scarcity of radio waves leads to congestion problems in wireless communication and this is overcome by the evolution of cognitive radio networks. The spectrum mobility phase involved in cognitive cycle plays a vital role by ensuring smooth handoff process. The existing methodologies proposed so far does not provide seamless connectivity and caters to the different network requirements hence the proposed work focuses on devising an intelligent method which administers and fine tunes the handoff process carried out by making decisions ahead of the change of ambience of network.

\section{PROPOSED SYSTEM}

The proposed work comprises of developing a cognitive mobile terminal which is capable of sensing the environment and establishing connections based on the availability of the primary user (PU) thereby resolves the problems caused by switching and the high interference rates occurring during data transmission[13]. The primary part in our work is using Genetic Algorithm (GA) with cuckoo search for handoff decision making, thereby optimizing fuzzy logic membership functions and which is new form of hybrid approach[14]. This work involves the usage of a new multi criteria kind of hybrid handoff strategy with cognitive ability to switch based on network conditions and availability of channel [15]. The flow of our proposed work is explained by means of Figure 1.

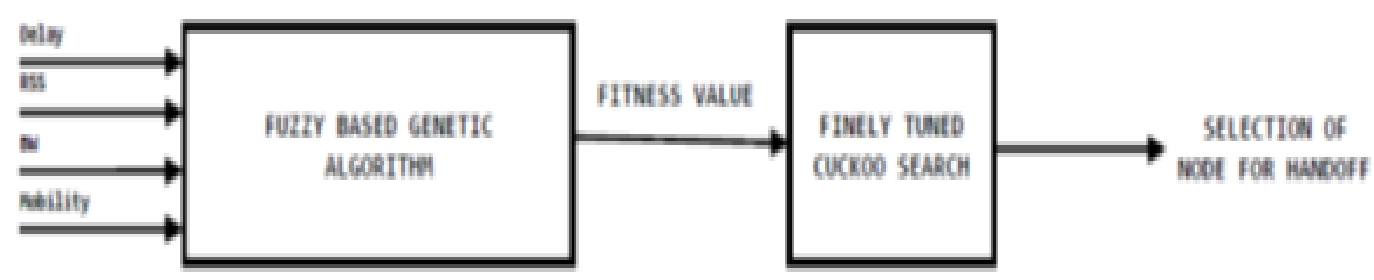

Figure 1. Flow of the proposed model

The proposed work can be split into two parts. The first part comprises of deploying the Fuzzy based Genetic Algorithm (FBGA) and subsequently incorporating cuckoo search for optimizing the output of the FBGA. FBGA gives the fitness value thereby yielding the selection of the appropriate channel to switch over based on the preference allocated. The procedure involved in FBGA and cuckoo search are described in the following section.

\subsection{Fuzzy Based Genetic Algorithm}

A FBGA is a hybrid form of GA which makes use of the fuzzy logic hinged procedure. The main purpose of using fuzzy logic is its convenient mode of addressing problems that are uncertain and unpredictable. The proposed method incorporates fuzzy logic in order to make decisions, which is an important part of the handoff process and this is achieved by making use of fuzzy logic controllers. 
Parameters that play vital role in handoff process are given as input to fuzzy inference which includes mobility, delay, received signal strength and bandwidth. These input parameters are then generated to if then else rules by means of the trapezoidal membership functions resulting in knowing the status of the PU that includes the arrival and departure of PU which is very important for deciding either the handoff process has to occur or not. The rules for generating handoff are presented in Table 1.

Table 1. Rules for Handoff Decision

\begin{tabular}{cccc}
\hline Distance & Speed & Delay & Handoff Priority \\
\hline Far & Fast & Low & VeryHigh \\
Far & Average & Low & VeryHigh \\
Far & Slow & Low & High \\
Medium & Fast & Medium & MedHigh \\
Medium & Average & Medium & MedHigh \\
Medium & Slow & Medium & MedHigh \\
Close & Fast & high & Med \\
Close & Average & high & MedLow \\
Close & Slow & high & MedLow \\
Far & Fast & Medium & VeryHigh \\
Far & Average & Medium & High \\
Far & Slow & Medium & MedHigh \\
Medium & Fast & Low & MedHigh \\
Medium & Average & Low & Med \\
Medium & Slow & Low & MedLow \\
Close & Fast & Medium & MedLow \\
Close & Average & Medium & Low \\
Close & Slow & Medium & VeryLow \\
Far & Fast & high & MedHigh \\
Far & Average & high & MedHigh \\
Far & Slow & high & Med \\
Medium & Fast & high & MedLow \\
Medium & Average & high & MedLow \\
Medium & Slow & high & MedLow \\
Close & Fast & Low & Low \\
Close & Average & Low & VeryLow \\
Close & Slow & Low & Verylow \\
\hline & & &
\end{tabular}

The trapezoidal membership functions are used to compute values for input parametes and in general they are given by

$$
\mu(x)=\left\{\begin{array}{cr}
1, & a 2<x<a 3 \\
0, & x=\text { a } 1 \text { or } a 4 \\
\frac{x-a 1}{a 2-a 1}, & a 1<x<a 2 \\
\frac{x-a 4}{a 3-a 4}, & a 3<x<a 4
\end{array}\right.
$$

From the above equation the membership functions for speed, distance and delay are computed separately. Speed membership Functions:

$$
\begin{gathered}
\mu \operatorname{low}(x)= \begin{cases}1, & 0<x<5 \\
0, & x=0 \text { or } 10 \\
\frac{x-0}{5-0}, & 0<x<5 \\
\frac{x-10}{7-10}, & 7<x<10\end{cases} \\
\mu \text { medium }(x)= \begin{cases}1, & 7<x<10 \\
0, & x=5 \text { or } x=15 \\
\frac{x-7}{10-7}, & 7<x<10 \\
\frac{x-15}{12-15}, & 12<x<15\end{cases}
\end{gathered}
$$




$$
\operatorname{\mu high}(x)=\left\{\begin{array}{lc}
1, & 15<x<20 \\
0, & x=12 \text { or } 25 \\
\frac{x-15}{12-15}, & 12<x<15 \\
\frac{x-25}{20-25}, & 20<x<25
\end{array}\right.
$$

Delay membership functions:

$$
\begin{gathered}
\mu l o w(x)=\left\{\begin{array}{lc}
1, & 0.1<x<0.2 \\
0, & x=0 \text { or } 0.25 \\
\frac{x-0}{0.1-0}, & 0<x<0.1 \\
\frac{x-0.2}{0.25-0.2}, & 0.2<x<0.25
\end{array}\right. \\
\mu \operatorname{medium}(x)=\left\{\begin{array}{lc}
1, & 0.3<x<0.4 \\
0, & x=0.2 \text { or } 0.5 \\
\frac{x-0.2}{0.3-0.2}, & 0.2<x<0.3 \\
\frac{x-0.5}{0.4-0.5}, & 0.4<x<0.5
\end{array}\right. \\
\mu h i g h(x)=\left\{\begin{array}{lc}
1, & 0.6<x<0.8 \\
0, & x=0.55 \text { or } 1.0 \\
\frac{x-0.55}{0.6-0.55}, & 0.55<x<0.6 \\
\frac{x-1.0}{0.8-1.0}, & 0.8<x<1.0
\end{array}\right.
\end{gathered}
$$

Distance membership functions:

$$
\begin{aligned}
& \mu \operatorname{low}(x)=\left\{\begin{array}{c}
1,2<x<15 \\
0, x=1 \text { or } 20 \\
\frac{x-15}{15-1}, 1<x<15 \\
\frac{x-20}{15-20}, 15<x<20
\end{array}\right. \\
& \mu \text { medium }(x)=\left\{\begin{array}{c}
1,12<x<50 \\
0, x=10 \text { or } 60 \\
\frac{x-10}{12-10}, 10<x<12 \\
\frac{x-60}{50-60}, 50<x<60
\end{array}\right. \\
& \mu h i g h(x)=\left\{\begin{array}{c}
1,60<x<80 \\
0, x=55 \text { or } 100 \\
\frac{x-55}{60-55}, 55<x<60 \\
\frac{x-100}{80-100}, 80<x<100
\end{array}\right.
\end{aligned}
$$

The inference obtained by fuzzy logic is partial, not accurate at all conditions and there is a need for GA to produce approximate results which includes availability of channel when PU and secondary user move across the network thereby enhancing the quality of handoff procedure. The GA gets terminated by iterating the algorithm till the suitable fitness value (FV) is generated. FV in the proposed work refers to the node predictability which specifies the values of input parameters associated with each and every single mobile node i.e. received signal strength, delay etc., indicating the mobility of the nodes connected to the network. The handoff priority is generated by means of the truth table presented in Table 1 . The value Distance represents the distance from mobile nodes to base station, Speed refers to the mobility of nodes and Delay are taken into consideration. The handoff decision is taken based on the truth table (table 1) containing the fuzzy rules. With respective to the distance variable the membership functions include far, medium, close and the 
range of values for these are $0.125,0.50$ and 0 respectively. In case of the variable speed the membership functions are slow, average, fast and the range of values are from 0 to 1 . The delay variable has membership functions that includes low, medium, high and the values are from $0.125,0.50$ and 0 respectively. The handoff priority is set to very high where the delay is low, the mobile nodes are far and the signal strength is fast. At this condition the handoff is initiated. The main functionalities carried out in FBGA includes initialization of population by real valued coding which is done by the selection of values using the roulette wheel, calculation of fitness values by means of membership functions and handoff resolution value. Upon the arrival of the PU which is determined by the fuzzy rules the other mobile nodes occupying the channel should vacate to avoid interference and this is achieved on the basis of using the access point candidate values stored in the handoff decision table. The decision of initializing the handoff is taken purely based on the access point candidate value obtained by fuzzy inference engine and the handoff resolution.

\subsection{Cuckoo Search}

The crux in choosing cuckoo search (CS) [16] is its simplicity and smooth implementation in comparison with other meta-heuristic algorithms. In context of our proposed work, cuckoo search is incorporated in reducing the occurrence of handoffs and selecting the optimal neighborhood before the handoff process which is the basic idea behind CS wherein the cuckoo bird searches for host nest to lay eggs [17]. FV obtained through FBGA mechanism is given as input to CS, determines the cases wherein the switch or change of state of mobile nodes in the network takes place. The procedure employed in fine-tuned CS includes the following steps:

Step 1: Consider a random population of $\mathrm{n}$ host nests as $\mathrm{xi}$

Step 2: A cuckoo is obtained randomly by levy flight behavior process $i$.

Step 3: The fitness function obtained by means of FBGA is taken as Fi.

Step 4: A random nest is chosen among the host nest $\mathrm{j}$ and its fitness is calculated as $\mathrm{Fj}$.

Step 5: If Fi $>\mathrm{Fj}$ then $\mathrm{j}$ is replaced by new solution else $\mathrm{j}$ is the solution.

Step 6: A fraction of the worst nest is eliminated and new nest are identified by means of levy flight search.

Step 7: The optimum nest is kept and step 2 is repeated for maximum iterations.

Step 8: The optimum nest is obtained finally.

Thus once new nest gets affixed it is evaluated by means of the previously computed FV thereby finding the ideal nest which turns out to be the best choice of determining which mobile node should undergo switching ahead of link failure based on channel state and this implies a condition of minimized handoff.

\section{RESULTS AND DISCUSSION}

The Network simulator NS 2.31 tool is used to carry out the simulation for the proposed work. Simulation is performed by ranging number of mobile nodes, initially the number of mobile nodes (MN) employed is 20; number of channels utilized is set to 10 and the maximum simulation time is set to 200 seconds. Once the switching takes place the spectrum manger senses the channel availability and initiates the next set of transmission. Figure 2 explains a case of simulation monitored at time $1.219490 \mathrm{~ms}$ wherein the MN 11 ends the handoff on channel 1 and the helper node is 8 for user 4 and the total number of packets sent is 9 and this process continues till MN 11 senses for channel and starts transmission at time $1.301000 \mathrm{~ms}$. Figure 3 explains yet another case of the simulation wherein transmission takes place across MN varying 4 to 8, 6 to 7 and so on. Packets sent during this period of time is from 18 to 100 packets dropped are also visible during the simulation period. This is again followed by the handoff, sensing and transmission phase till the simulation gets over.

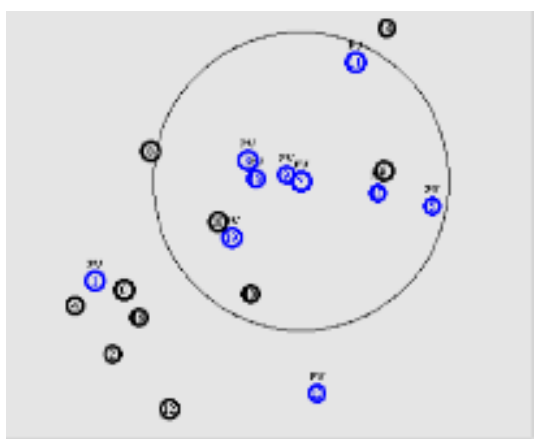

Figure 2. Handoff temination

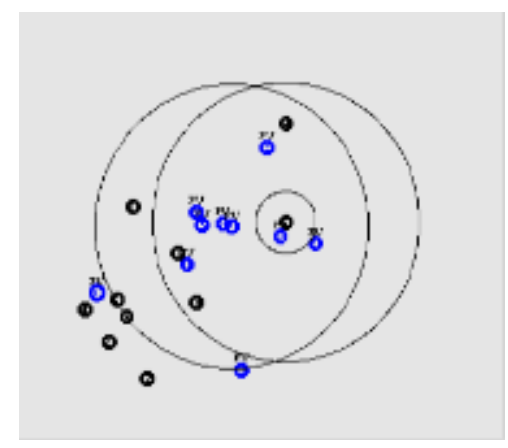

Figure 3. Switching of interface 
Based on the simulation carried out the analysis of handoff parameters i.e. the factors that overall determines the quality of handoff can be monitored easily. The parameters taken for consideration include throughput, delay and number of handoffs occurred and number of failed handoff. These are discussed briefly in the following section.

a. Throughput: This parameter represents the successful transmission of data over the given period of time from source to destination it is the benchmark for deciding the overall efficiency of the system. Figure 4.represents the amount of data transmission occurred during the simulation time with $\mathrm{X}$ axis representing simulation time in seconds and $\mathrm{Y}$ axis representing the amount of data transmitted in kbps.

b. Delay: This depicts the time taken for transmission of bits from source node to destination node. Figure 5. represents the overall delay occurred during the handoff process with $\mathrm{X}$ axis representing the simulation time in seconds and Y axis representing the delay period in seconds.

c. Number of handoffs: The maximum number of times switching of states occur during data transmission refers to as the number of handoff parameter. This should not take place for more number of times. Figure 6 represents the number of handoff process occurred during the entire simulation time with $\mathrm{X}$ axis representing simulation time in seconds and $\mathrm{Y}$ axis representing the number of handoff process occurred with two lines representing the existing and proposed system. From the simulation results it is clear that the proposed system changes its states minimal number of times compared to the existing system.

d. Number of failed handoffs: This gauge exhibits the capacity of a network i.e. indicates cases wherein a state in a channel during transmission is unsuccessful. Figure 7. represents the number of failed handoffs occurred during the simulation time with $\mathrm{X}$ axis representing simulation time in seconds and $\mathrm{Y}$ axis representing the number of failed handoff process.

From the analysis of the parameters involved in the handoff process generated and the comparison of handoff schemes generated by dynamic programming with bisection (DBA) algorithm, fuzzy based genetic and the proposed FBGA with CS in Table 2, evident that the results are far better and are optimized compared to the existing systems in terms of uncertain network conditions.

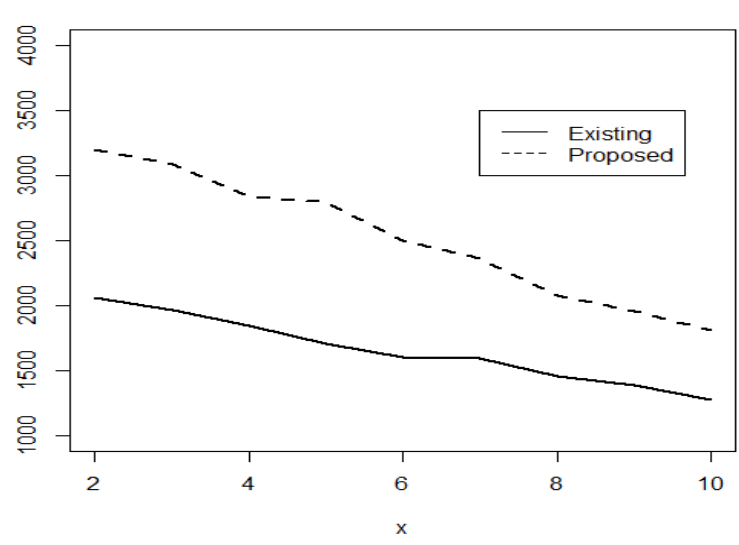

Figure 4. Throughput

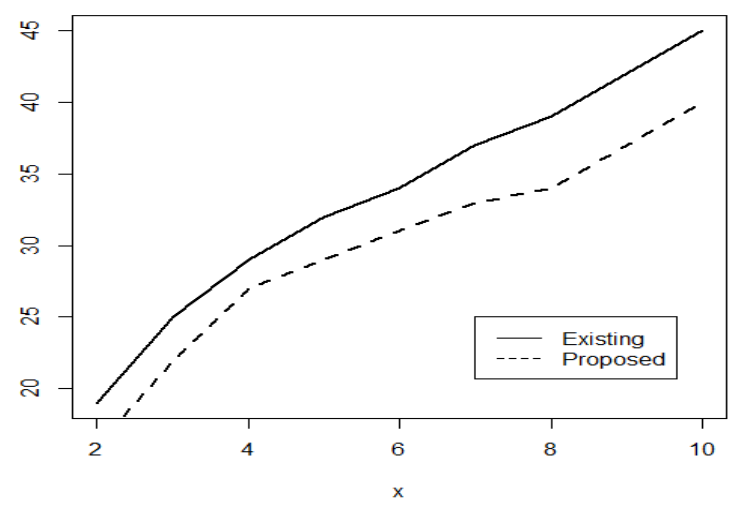

Figure 6. Number of handoffs

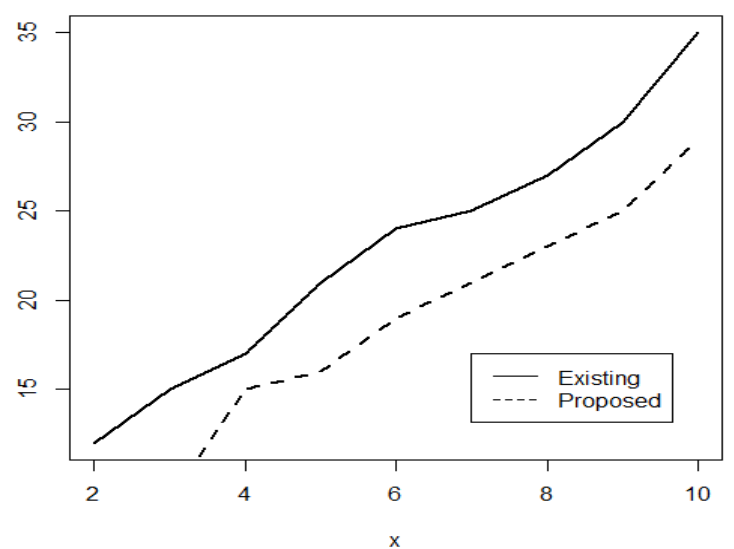

Figure 5. Delay

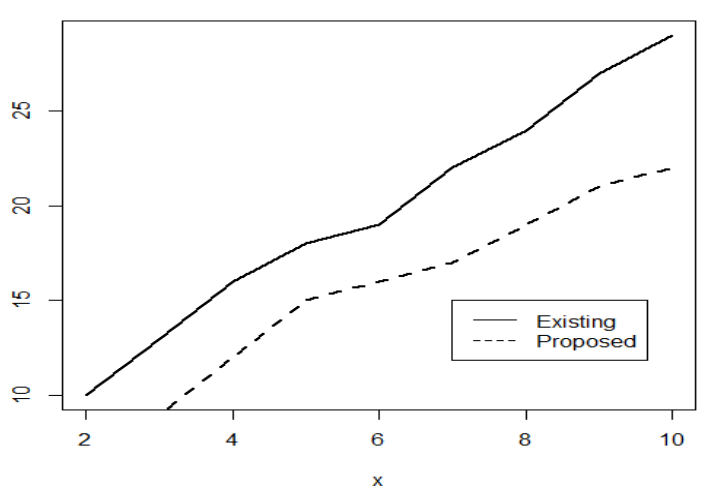

Figure 7. Number of failed handoffs 
Table 2. Comparison of Handoff Schemes

\begin{tabular}{|c|c|c|c|c|}
\hline Handoff Parameters & $\begin{array}{c}\text { Transmission time in } \\
\text { sec }\end{array}$ & $\begin{array}{c}\text { Handoff based on } \\
\text { DBA }\end{array}$ & $\begin{array}{c}\text { Handoff } \\
\text { based on } \\
\text { FGA } \\
\end{array}$ & $\begin{array}{c}\text { Proposed } \\
\text { Handoff based on } \\
\text { hybrid FBGA with CS }\end{array}$ \\
\hline \multirow{10}{*}{ Average Delay in sec } & 1 & 12 & 8 & 4 \\
\hline & 2 & 16 & 12 & 7 \\
\hline & 3 & 19 & 15 & 9 \\
\hline & 4 & 21 & 17 & 15 \\
\hline & 5 & 23 & 21 & 16 \\
\hline & 6 & 26 & 24 & 19 \\
\hline & 7 & 28 & 25 & 21 \\
\hline & 8 & 31 & 27 & 23 \\
\hline & 9 & 32 & 30 & 25 \\
\hline & 10 & 38 & 35 & 29 \\
\hline \multirow{11}{*}{$\begin{array}{l}\text { Average Throughput } \\
\text { in kbps }\end{array}$} & 1 & 1892 & 2174 & 3512 \\
\hline & 2 & 1750 & 2058 & 3201 \\
\hline & 3 & 1627 & 1964 & 3085 \\
\hline & 4 & 1582 & 1846 & 2840 \\
\hline & 5 & 1488 & 1702 & 2792 \\
\hline & 6 & 1201 & 1600 & 2502 \\
\hline & 7 & 1057 & 1592 & 2359 \\
\hline & 8 & 937 & 1458 & 2075 \\
\hline & 9 & 893 & 1387 & 1957 \\
\hline & 10 & 742 & 1272 & 1810 \\
\hline & 1 & 24 & 18 & 14 \\
\hline \multirow{8}{*}{ Number of Handoffs } & 2 & 26 & 19 & 16 \\
\hline & 3 & 31 & 25 & 22 \\
\hline & 4 & 34 & 29 & 27 \\
\hline & 5 & 38 & 32 & 29 \\
\hline & 6 & 42 & 34 & 31 \\
\hline & 7 & 44 & 37 & 33 \\
\hline & 8 & 49 & 39 & 34 \\
\hline & 9 & 52 & 42 & 37 \\
\hline \multirow{11}{*}{$\begin{array}{c}\text { Number of Failed } \\
\text { Handoffs }\end{array}$} & 10 & 55 & 45 & 40 \\
\hline & 1 & 10 & 7 & 4 \\
\hline & 2 & 13 & 10 & 7 \\
\hline & 3 & 15 & 13 & 9 \\
\hline & 4 & 19 & 16 & 12 \\
\hline & 5 & 22 & 18 & 15 \\
\hline & 6 & 26 & 19 & 16 \\
\hline & 7 & 28 & 22 & 17 \\
\hline & 8 & 31 & 24 & 19 \\
\hline & 9 & 34 & 27 & 21 \\
\hline & 10 & 37 & 29 & 22 \\
\hline
\end{tabular}

\section{CONCLUSION}

In this paper, the handoff process a part of the spectrum mobility a challenging task in CRN is addressed and analysed by incorporating hybrid methodology of fuzzy based genetic algorithm and it is optimised using cuckoo search. The simulation results show that the proposed method is reliable one and ensures that the handoff process is carried out in a smooth way with minimal backlogs in comparison with the existing system.

\section{REFERENCES}

[1] Hernández, C.; Salgado, C.; López, H.; Rodríguez-Colina, E., "Multivariable algorithm for dynamic channel selection in cognitive radio networks," EURASIP Journal on Wireless Communications and Networking, vol. 2015, no. 1, pp. 1-17, 2015.

[2] Krishan Kumar, Arun Prakash and Rajeev Tripathi, "Spectrum Handoff Scheme with ultiple Attributes Decision Making for Optimal Network Selection in Cognitive Radio Networks", Digital Communications and Networks,January 2017.

[3] Pradip Varade,Akanksha Wabale, "Throughput Maximization of Cognitive radio multi relay network with interference management", International Journal of Electrical and Computer Engineering (IJECE), Vol. 8, No. 4, August 2018, pp. 2230-2238. [4] PrithiviRaj A, Krishnamurthy K, "Fuzzy logic based decision making algorithm to optimize the handoff performance in Hetnets", Circuits and Systems 756-3777, September 2016.

[4] PrithiviRaj A, Krishnamurthy K, "Fuzzy logic based decision making algorithm to optimize the handoff performance in Hetnets”, Circuits and Systems 756-3777, September 2016. 
[5] Preetham C.S, Prasad M.S.G et al, "Performance analysis of cooperative hybrid Cognitive radio network with various diversity techniques", International Journal of Electrical and Computer Engineering (IJECE), Vol.6, No.5, October 2016, pp 2125-2133.

[6] Tsai, K., Liu, H. and Liu, Y, "Using Fuzzy Logic to Reduce Ping Pong Handover Effect in LTE Networks”, Soft Computing, 20, 1683-1694, 2016.

[7] Potdar, S.M.; Patil, K.P., "Efficient spectrum handoff in CR network based on mobility, QoS and priority using fuzzy logic and neural network," Contemporary Computing (IC3), 2013 Sixth International Conference on , vol., no., pp.53,58, 8-10 Aug. 2013.

[8] Prudhvi Raj Metti, K. Rushendra Babu, Sumit Kumar, "Spectrum Handoff Mechanism in Cognitive Radio Networks using Fuzzy Logic" International Journal of Scientific \& Engineering Research, Volume 5, Issue 10, October-2014.

[9] Wanmai Yuan, Mingchuan Yang, "Improved cuckoo search algorithm for spectrum sensing in sparse satellite cognitive systems" IEEE $84^{\text {th }}$ Vehicular Technology conference 2016.

[10] Ali Calhan, Celal Cekan "An Optimum Vertical Handoff Decision Algorithm Based on Adaptive Fuzzy Logic and Genetic Algorithm”, Wireless Pers Commun (2012) 64:647-664.

[11] Miguel Tuberquia, Cesar Hernandez, "New Approaches in cognitive radios using evolutionary algorithms", International journal of Electrical and Computer Engineering (IJECE),Vol. 8, No. 3, June 2018, pp. 1636-1646.

[12] Kale Sandikar R.S, Vijay M.Wadhai, "New Algorithm for Handoff Optimization in Cognitive Radio Networks using Fuzzy logic and Artificial Neural Network", Elsevier Publications,2013.

[13] Salgado.C, Hernandez.C, Molina.V, "Intelligent algorithm for spectrum mobility in cognitive wireless networks", Procedia Computer Science 83, 278 - 283, 2016.

[14] Ekta Dua, Vikrant Gulati, "Enhancement in Spectrum Sensing Algorithms for Cognitive Radios” International Journal of Advanced Research in Electronics and Communication Engineering (IJARECE) Volume 5, Issue 8, August 2016.

[15] hang Trung Nguyen, Dieu Ngoc Vo, "The application of one rank cuckoo search algorithm for solvingeconomic load dispatch problems" Applied Soft Computing 37, 763-773,2015.

[16] W. Ahmed, J. Gao and H. Suraweera, "Analysis of cognitive radio spectrum access with optimal channel reservation", IEEE Transactions on Wireless Communications, vol. 8, no. 9, pp. 4488-4491,2014.

[17] Yanhong Feng, Ke Jia, and Yichao He, "An Improved Hybrid Encoding Cuckoo SearchAlgorithm for 0-1 Knapsack Problems", Hindawi Publishing Corporation Computational Intelligence and Neuroscience Volume 2014.

\section{BIOGRAPHIES OF AUTHORS}
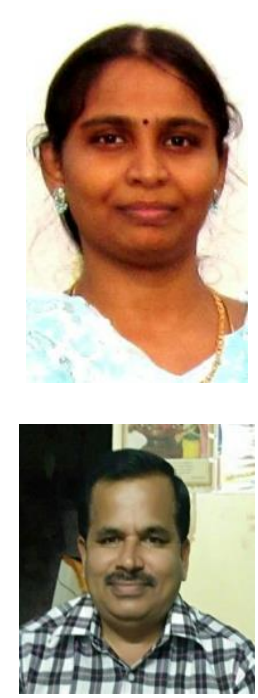

J.Josephine Dhivya received the MCA degree from Madurai Kamaraj University and is currently a Research Scholar in Madurai Kamaraj University.Her areas of interest include Wireles Communications and Networking
Dr. M. Ramaswami (M.Sc, M.Phil, M.C.A) was awarded the Phd degree in Computer Applications from Madurai Kamaraj University. He is currently working as a Professor in the Department of Computer Applications in Madurai Kamaraj University and an active research supervisor and he has published about 45 papers in various reputed journals at national and international level. 